\section{Novel 6-Month Treatment for Drug-Resistant Tuberculosis, United States}

\author{
Connie A. Haley, Patricia Macias, Supriya Jasuja, \\ Betsy A. Jones, Marie-Claire Rowlinson, \\ Roshni Jaimon, Pennelyn Onderko, Elaine Darnall, \\ Maria E. Gomez, Charles Peloquin, David Ashkin, \\ Neela D. Goswami
}

Author affiliations: University of Florida, Gainesville, Florida, USA (C.A. Haley, M.E. Gomez, D. Ashkin); Cook County Department of Public Health, Chicago, Illinois, USA (P. Macias, S. Jasuja, R. Jaimon, P. Onderko); Florida Department of Health, Jacksonville, Florida, USA (B.A. Jones, M.-C. Rowlinson, D. Ashkin) Illinois Department of Public Health, Springfield, Illinois, USA

(E. Darnall); University of Florida College of Pharmacy, Gainesville (C. Peloquin); Centers for Disease Control and Prevention, Atlanta, Georgia, USA (N.D. Goswami)

\section{DOI: https://doi.org/10.3201/eid2701.203766}

The US Food and Drug Administration approved a 6-month regimen of pretomanid, bedaquiline, and linezolid for extensively drug-resistant or multidrug-intolerant tuberculosis after a trial in South Africa demonstrated $90 \%$ effectiveness 6 months posttreatment. We report on a patient who completed the regimen using a lower linezolid dose.

$\mathrm{A}^{2}$ woman from eastern Europe in her late 40s undergoing treatment for stage III cervical adenocarcinoma was found to have a right upper lobe pulmonary nodule. Pathology from tissue biopsy demonstrated necrotizing granulomas and numerous acid-fast bacilli (AFB); the sample was PCR positive for Mycobacterium tuberculosis. Adjuvant chemotherapy was held, and the patient was referred to the local public health department. The patient provided informed written consent for publication of her case study.

On evaluation, the patient was asymptomatic without physical findings and reported no previous diagnosis or treatment of tuberculosis (TB) disease or latent TB infection. Results of serologic testing for HIV and viral hepatitis B and C were negative. She had a mild chronic anemia and transient transaminitis during chemotherapy (peak alanine aminotransferase $215 \mathrm{IU} / \mathrm{L}$; aspartate aminotransferase 185 IU/L). Three sputum samples were negative by AFB smear and culture; 1 was tested by PCR and was $M$. tuberculosis negative.

Treatment with rifampin, isoniazid, pyrazinamide, ethambutol and pyridoxine was initiated.
Lung biopsy cultures grew $M$. tuberculosis, and GeneXpert MTB/RIF assay (Cepheid, https://www.cepheid.com) detected an rpoB mutation indicating likely rifampin resistance. Rapid molecular detection of drug resistance and growth-based drug susceptibility testing performed by the Centers for Disease Control and Prevention (CDC) and Florida Bureau of Public Health Laboratories yielded concordant results. We detected resistance to isoniazid, rifampin, the fluoroquinolones (levofloxacin and moxifloxacin), and an injectable (kanamycin), confirming a diagnosis of extensively drug-resistant TB (XDR TB). Resistance was also detected for pyrazinamide but not for ethambutol, bedaquiline, or linezolid. The patient and her medical providers, in consultation with a CDCfunded TB Center of Excellence (COE, https:/ / www. cdc.gov/tb/education/tb_coe), determined that her best treatment option was a 6-month all-oral regimen of bedaquiline, pretomanid, and linezolid (BPaL).

$\mathrm{BPaL}$ was approved by the US Food and Drug Administration (FDA) on August 14, 2019, based in part on results from the Nix-TB trial in South Africa, which included patients with XDR TB or multidrugresistant (MDR) TB who failed or were intolerant of prior therapy (1). Pretomanid, the novel agent in the regimen, is a nitroimidazooxazine that blocks cellwall production in actively replicating MTB organisms and acts as a respiratory poison and protein synthesis inhibitor to kill nonreplicating persister organisms (2). Bedaquiline is a diarylquinoline that inhibits mycobacterial adenosine triphosphate synthase in replicating and persister organisms, and linezolid is an oxazolidinone that also inhibits protein synthesis $(3,4)$. The combined activity of $\mathrm{BPaL}$ enables cure in a far shorter period compared with currently recommended 18- to 24-month MDR TB regimens (5). In the Nix-TB trial, $\mathrm{BPaL}$ produced favorable outcomes in 98/109 (90\%) patients at 6 months posttreatment (1); in addition, little preexisting resistance to bedaquiline, pretomanid, or linezolid has been reported $(4,6)$.

Because pretomanid was not yet commercially available in the United States, the TB Alliance required an FDA-approved single-patient investigational new drug application and provided 6 months of pretomanid acquired internationally. A bridging regimen of bedaquiline, linezolid, moxifloxacin, cycloserine, clofazimine, and ethambutol was initiated for 2 weeks, then was narrowed to BPaL when pretomanid arrived.

For this patient, we initiated linezolid at $600 \mathrm{mg} / \mathrm{d}$, given the toxicity of the Nix-TB dose of 1,200 mg/d, the patient's paucibacillary disease, and TB COE's 
Table 1. Molecular susceptibility sequencing results and therapeutic drug monitoring data from treatment of XDR TB, Florida, USA*

\begin{tabular}{|c|c|c|c|c|c|c|}
\hline Drug (dose) & Sequencing result & $\begin{array}{l}\text { Date drug } \\
\text { level drawn }\end{array}$ & Trough, $\mu \mathrm{g} / \mathrm{mL}$ & $\begin{array}{l}\text { 2h postdose } \\
\text { level, } \mu \mathrm{g} / \mathrm{mL}\end{array}$ & $\begin{array}{l}\text { 6h postdose } \\
\text { level, } \mu \mathrm{g} / \mathrm{mL}\end{array}$ & $\begin{array}{l}\text { Typical peak serum } \\
\text { concentration, } \mu \mathrm{g} / \mathrm{mL}\end{array}$ \\
\hline $\begin{array}{l}\text { Bedaquiline (200 mg } \\
\text { MWF) }\end{array}$ & $\begin{array}{c}\text { No atpE (ORF) } \\
\text { mutation detected; no } \\
\text { Rv0678/mmpR (ORF) } \\
\text { mutation detected }\end{array}$ & 2019 Nov 13 & $\begin{array}{l}0.51(42.25 \mathrm{~h} \\
\text { postdose })\end{array}$ & 1.40 & 1.42 & $\begin{array}{c}1.2-1.8 \\
(5-6 \mathrm{~h} \text { postdose, } \\
\text { maintenance phase) }\end{array}$ \\
\hline $\begin{array}{l}\text { N-monodesmethyl } \\
\text { bedaquiline } \\
\text { (metabolite) }\end{array}$ & NT & 2019 Nov 13 & $\begin{array}{l}0.22(42.25 \mathrm{~h} \\
\text { postdose })\end{array}$ & 0.24 & 0.27 & NT \\
\hline Pretomanid $(200 \mathrm{mg} / \mathrm{d})$ & NT & 2019 Nov 13 & $\begin{array}{l}2.07(18.25 \mathrm{~h} \\
\text { postdose })\end{array}$ & 3.43 & 2.98 & $\begin{array}{c}2.3-4.3 \\
\text { (5-6 h postdose, at } \\
\text { steady state) }\end{array}$ \\
\hline Linezolid (600 mg/d) & $\begin{array}{l}\text { No rplC (ORF aa } 84- \\
217) \text { mutation detected; } \\
\text { no rrl (nt 2191-2929) } \\
\text { mutation detected }\end{array}$ & 2019 Nov 13 & $\begin{array}{l}7.62(18.25 \mathrm{~h} \\
\text { postdose })\end{array}$ & 24.15 & 17.88 & $12-26$ \\
\hline $\begin{array}{l}\text { Linezolid (600 mg } \\
\text { MWF) }\end{array}$ & NT & 2020 Mar 12 & $<2.00 \dagger$ & 19.04 & 13.6 & $12-26$ \\
\hline
\end{tabular}

*MWF, Monday/Wednesday/Friday; NT, not tested; ORF, open reading frame; XDR, extensively drug-resistant tuberculosis.

$\dagger$ Trough sample was not collected; based on the apparent elimination half-life, the linezolid concentration at $48 \mathrm{~h}$ was calculated to be $<2 \mu \mathrm{g} / \mathrm{mL}$, a value associated with minimal toxicity.

experience with linezolid dosing $(1,4,7,8)$. Therapeutic drug monitoring performed at the University of Florida Infectious Diseases Pharmacokinetic Laboratory (https://idpl.pharmacy.ufl.edu) was used to maintain a linezolid peak of $12-26 \mu \mathrm{g} / \mathrm{mL}$ and trough $<2 \mu \mathrm{g} / \mathrm{mL}$ to reduce drug-induced toxicity $(4,9)$.

The patient received outpatient $\mathrm{BPaL}$ treatment 7 days a week by directly observed therapy. We assessed liver, renal, hematologic, and neurologic function plus QTC intervals at baseline and every 2-4 weeks during treatment (Table 1). A few weeks into therapy, the patient's linezolid level 18 hours postdose was measured at $7.62 \mu \mathrm{g} / \mathrm{mL}$ (serum trough level at 24 hours was likely lower but was still higher than expected) (Table 2, https://wwwnc.cdc.gov/EID/ article/27/1/20-3766-T2.htm). To reduce the trough while maintaining a peak serum level 4-16 times over her M. tuberculosis isolate's linezolid MIC of $0.12 \mu \mathrm{g} /$ $\mathrm{mL}$, we extended the linezolid dosing interval to 600 mg every Monday, Wednesday, and Friday. A subsequent linezolid trough at 48 hours was calculated at $<2 \mu \mathrm{g} / \mathrm{mL}$. The patient completed 182 doses of $\mathrm{BPaL}$ over 26 weeks without treatment interruptions. Other than mild nausea that responded to pantoprazole, she had no adverse events or notable changes in laboratory values or electrocardiographs. Nine months after completion, the patient remained well; the state health department expects to closely monitor her for recurrent TB for 24 months after BPaL completion.

The patient, physicians, and public health staff involved reported high satisfaction with BPaL. Providers and TB programs in the United States considering this regimen for TB patients can seek guidance from $C D C$ Division of Tuberculosis Elimination or their TB COE.
Current trials using BPaL, suchas ZeNix (https:/ / www. tballiance.org/portfolio/trial/11883), are evaluating lower doses and shorter duration of linezolid compared with those of the Nix-TB trial. The 6-month, all oral, highly effective $\mathrm{BPaL}$ regimen is a notable advancement toward reducing global TB deaths (10).

\section{About the Author}

Dr. Haley is a medical consultant for the Southeast National TB Center and an adjunct clinical professor in the Department of Medicine, Division of Infectious Diseases and Global Medicine, University of Florida.

\section{References}

1. Conradie F, Diacon AH, Ngubane N, Howell P, Everitt D, Crook AM, et al.; Nix-TB Trial Team. Treatment of highly drug-resistant pulmonary tuberculosis. N Engl J Med. 2020;382:893-902. https:// doi.org/10.1056/NEJMoa1901814

2. Keam SJ. Pretomanid: first approval. Drugs. 2019;79:1797803. https:// doi.org/10.1007/s40265-019-01207-9

3. Lee M, Lee J, Carroll MW, Choi H, Min S, Song T, et al. Linezolid for treatment of chronic extensively drug-resistant tuberculosis. N Engl J Med. 2012;367:1508-18. https://doi.org/10.1056/NEJMoa1201964

4. Brown AN, Drusano GL, Adams JR, Rodriquez JL, Jambunathan K, Baluya DL, et al. Preclinical evaluations to identify optimal linezolid regimens for tuberculosis therapy. MBio. 2015;6:e01741-15. https:/ / doi.org/10.1128/ mBio.01741-15

5. Nahid P, Mase SR, Migliori GB, Sotgiu G, Bothamley GH, Brozek JL, et al. Treatment of drug-resistant tuberculosis. An official ATS/CDC/ERS/IDSA clinical practice guideline. Am J Respir Crit Care Med. 2019;200:e93-142. https://doi.org/10.1164/rccm.201909-1874ST

6. Xu J, Wang B, Hu M, Huo F, Guo S, Jing W, et al. Primary clofazimine and bedaquiline resistance among isolates from patients with multidrug-resistant tuberculosis. Antimicrob Agents Chemother. 2017;61:e00239-17. 
https://doi.org/10.1128/AAC.00239-17

7. Peloquin $\mathrm{C}$. The role of therapeutic drug monitoring in mycobacterial infections. Microbiol Spectr. 2017;5. https://doi.org/10.1128/microbiolspec.TNMI7-0029-2016

8. Heinrichs MT, Drusano GL, Brown DL, Maynard MS, Sy SKB, Rand KH, et al. Dose optimization of moxifloxacin and linezolid against tuberculosis using mathematical modeling and simulation. Int J Antimicrob Agents. 2019;53:275-83. https://doi.org/10.1016/j.ijantimicag.2018.10.012

9. Song T, Lee M, Jeon HS, Park Y, Dodd LE, Dartois V, et al. Linezolid trough concentrations correlate with mitochondrial toxicity-related adverse events in the treatment of chronic extensively drug-resistant tuberculosis. EBioMedicine. 2015;2:1627-33. https:/ / doi.org/10.1016/ j.ebiom.2015.09.051

10. Harding E. WHO global progress report on tuberculosis elimination. Lancet Respir Med. 2020;8:19. https:/ / doi.org/ 10.1016/S2213-2600(19)30418-7

Address for correspondence: Connie A. Haley, Southeastern

National Tuberculosis Center, Box 103600, Gainesville, FL 326103600, USA; email: connie.haley@medicine.ufl.edu

\section{COMMENT LETTERS}

\section{Large-Scale Isolation Facilities and Potential for Secondary Infectious Disease Outbreak}

\author{
Shi Yu Derek Lim, ${ }^{1}$ Hong Liang Tey ${ }^{1}$ \\ Author affiliations: National Skin Centre, Singapore (S.Y.D. Lim, \\ H.L. Tey); Woodlands Health Campus, Singapore (S.Y.D. Lim, \\ H.L. Tey); National University of Singapore (H.L. Tey); Nanyang \\ Technological University, Singapore (H.L. Tey)
}

DOI: https://doi.org/10.3201/eid2701.203127

To the Editor: Singapore has instituted large-scale isolation facilities similar to those detailed by Choi et al. (1) for patients with mild coronavirus disease. We highlight the risk for transmission of secondary infectious diseases by sharing our experience with a varicella outbreak.

Three patients, all migrant workers housed in the same isolation hall, were seen for vesicular eruptions, later laboratory confirmed as varicella, within the span of 9 days. The first patient's symptoms were truncal erythematous-based vesicles and erosions after a prodrome of fever and headache. He was promptly transferred for further hospital isolation. As part of a ring vaccination strategy, we offered 200 close contacts postexposure vaccination. However, 2 other patients, not close contacts of the first, had similar eruptions; for the second patient, 7 days later with a rash duration of 2 days, and for the third, 8 days after, with a rash duration of 6 days (Figure). After these additional cases, vaccination was offered to all remaining patients in the isolation facility.

\footnotetext{
${ }^{1}$ These authors contributed equally to this article.
}

All 3 patients probably contracted varicella from unidentified persons with varicella or zoster infection, given that illness onset fell short of the usual 10-21-day incubation period (2). Although varicella seroprevalence among adults in Singapore is high $(88 \%)$, data on seroprevalence among migrant workers remain limited (3).

Although isolation facilities obviate the capacity constraints of hospital isolation, our experience highlights the potential for secondary outbreaks, which are disruptive and costly to investigate and control. To mitigate this risk, preentry screening inquiring about previous chickenpox infection or vaccination should be considered. Serologic screening is ideal but challenging to implement. Among patients, social distancing and face coverings should be enforced. We also recommend active surveillance for vesicular rash and fever, prompt isolation of patients with suspected cases, and vaccination of identified close contacts without previous infection, vaccination, or contraindications to vaccination, as

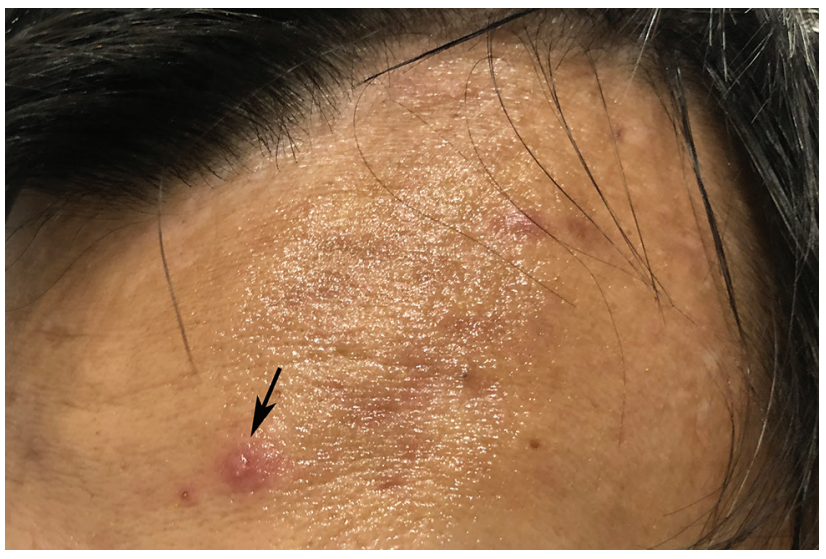

Figure. Vesicle on an erythematous base (arrow), commonly described as "dewdrop on rose petal", over the forehead of a patient with varicella, Singapore, 2020. 